\title{
Der schwer zu sedierende Patient
}

\author{
Christian Ertmer
}

\section{Einleitung zur Rubrik}

In dieser Rubrik werden Experten, die die aktuelle Literatur sehr gut überblicken und umfassende klinische Erfahrung haben, Antworten auf Ihre Fragen liefern, die sich nicht so einfach aus der Literatur beantworten lassen. Wenn Sie also eine außergewöhnliche Frage haben, auf die Sie sich schon lange eine Antwort wünschen, freuen wir uns auf Ihre Zuschrift an Gabi.Hasenmaile@thieme.de.

\section{Ask the Expert}

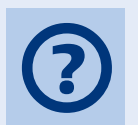

Welche Therapieoptionen stehen für „schwer sedierbare Patienten“ zur Verfügung, die selbst mit einer Vierfachfachkombination an Sedativa (Midazolam, Sufentanil, Ketamin,

Propofol) plus Clonidin nicht ausreichend tief zu sedieren sind? Ist Methohexital eine mögliche Substanz?

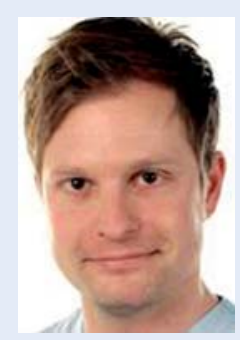

Christian Ertmer

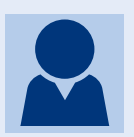

Bei jedem sedierten Patienten sollte mindestens einmal täglich kritisch die Indikation zur Sedierung per se und (bei gegebener Indikation) die angestrebte Sedierungstiefe reevaluiert werden. Nur wenige Patienten benötigen eine sehr tiefe Sedierung (RASS-4/-5). In der Klinik des Verfassers trifft dies nahezu ausschließlich auf Patienten mit instabiler zerebraler Zirkulation bzw. erhöhtem intrakraniellen Druck zu. Gerade bei diesen, oft jungen Patienten ist eine tiefe Sedierung jedoch häufig sogar unter differenzierten Medikamentenkombinationen nur schwer zu erreichen.

Wenn unter einer Kombination aus Sedativum (Propofol oder Midazolam) und Opioid (Sufentanil oder Fentanyl) keine hinreichende Sedierung erreicht wird, kommt häufig S-Ketamin zum Einsatz und wird auch in der S3-Leitlinie „Analgesie, Sedierung und Delirmanagement in der Intensivmedizin“ in dieser Indikation empfohlen (Empfehlungsgrad B). Alle über diese Kombination hinausgehenden Erweiterungen der Sedie- rung sind in Auslegung der Leitlinie als „off-label“ zu bezeichnen.

Im klinischen Alltag hat sich die supportive Infusion eines alpha-2-Agonisten wie Clonidin bewährt. Die Datenlage für Dexmedetomidin ist in der Anästhesiologie bei neurochirurgischen Eingriffen vielversprechend, in der Intensivmedizin in dieser Indikation (tiefe Sedierung) aber noch unzureichend dokumentiert. Dennoch kann (als individueller Heilversuch) nach Erfahrung des Verfassers ein Versuch mit Dexmedetomidin bei schwer zu sedierenden Patienten unternommen werden.

Ein Wechsel des Opioids von Sufentanil/Fentanyl auf Remifentanil kann in Einzelfällen einen erheblichen Effekt auf die Sedierungstiefe haben.

Sehr effektiv ist die Anwendung volatiler Anästhetika (Sevofluran, Isofluran) bei schwer zu sedierenden Patienten. Allerdings ist dies nur im Rahmen individueller Heilversuche zulässig und bei Patienten mit erhöhtem intrakraniellen Druck weiterhin formal kontraindiziert.

Als potenziell vielversprechend wird das kurzwirksame, nicht kumulierende Benzodiazepin Lormetazepam propagiert. Allerdings existieren bislang nur unzureichende Daten zur Verwendung dieser Substanz, insbesondere in Bezug auf das delirogene Potenzial und die Langzeiteffekte.

Barbiturate sollten (nach Meinung des Verfassers) nur in äußerst seltenen Einzelfällen verwendet werden. In der Klinik des Verfassers sind dies ausschließlich Fälle von refraktär erhöhtem intrakraniellen Druck mit per se hoher Letalitätswahrscheinlichkeit. Eine Sedierung mit Barbituraten von Patienten ohne SchädelHirn-Verletzung sollte nicht erfolgen. 
Zu Methohexital existiert in seiner Anwendung als Langzeitsedativum sehr wenig Literatur. Eine deutsche, nicht randomisierte Arbeit mit kleiner Fallzahl sieht Vorteile bezüglich der Delirentstehung (Volz et al., Anaesthesist. 2014 Jun; 63 (6): 488 -95). Es scheint pharmakokinetisch Vorteile gegenüber dem Thiopental zu bieten bei ähnlichen Eigenschaften z. B. auf den intrakraniellen Druck. Daher wird es vor allem in der Pädiatrie nicht selten für kurze Prozeduren (z.B. Intubation oder Katheteranlagen/-entfernungen) eingesetzt. Die Diskussion um die unerwünschten Wirkungen der Barbiturate, hier vor allem die Beeinflussung des Immunsystems, ist besorgniserregend.

In Ermangelung guter prospektiver Daten zu Methohexital als Langzeitsedativum kann der Einsatz vor dem Hintergrund gut verfügbarer und untersuchter Alternativen nicht empfohlen werden.

Sollte das Problem nicht primär die Sedierung, sondern die Reflexantwort z. B. auf Lagerungswechsel oder Absaugen sein, muss eine Muskelrelaxation für kürzere Zeit, vorzugsweise mit Cisatracurium, erwogen werden.

Interessenkonflikt: Der Autor gibt an, dass kein Interessenkonflikt besteht.

\section{Korrespondenzadresse}

Priv.-Doz. Dr. med. Christian Ertmer

Universitätsklinikum Münster

Klinik für Anästhesiologie, operative Intensivmedizin und Schmerztherapie

Albert-Schweitzer-Campus 1, Gebäude A1

48149 Münster

ertmer@anit.uni-muenster.de 